Rev. Elev. Méd. vét. Pays trop., 1970, 23 (3) : 333-35

\title{
Note sur quelques modalités de l'insémination chez les glossines (diptera-muscidae)
}

\author{
par R. TIBAYRENC et J. ITARD
}

\begin{abstract}
RESUME
La présente note confirme en lui apportant certaines précisions, l'observation faite par J. N. POLLOCK sur Glossina austeni Newstead : le transport du sperme au cours du rapprochement sexuel s'effectue par l'intermédiaire d'un «spermatophore », qui se constitue chez le mâle avant d'être introduit dans l'utérus de la femelle.
\end{abstract}

A la suite de l'observation publiée par J. N. POLLOCK en mars de cette année, fut entreprise une étude systématique du transport de la semence au cours de l'accouplement chez les Glossines.

Travaillant d'abord sur Glossina austeni Newstead, on observa très facilement la présence d'un "spermatophore " dans l'utérus de la femelle une fois l'accouplement terminé, c'est-à-dire lorsque les deux sexes se sont séparés (la durée du rapprochement est variable, d'une durée minimale d'une heure et demie) (photo $\mathrm{n}^{\circ}$ 1). Glossina tachinoides Westwood et Glossina morsitans Westwood présentent également un tel "spermatophore " (l'accouplement étant en général assez bref pour la première espèce et beaucoup plus long pour la deuxième, de plusieurs heures).

Ce « spermatophore » est une masse globuleuse assez irrégulière, de consistance muqueuse, dont les axes mesurent plusieurs centaines de microns (autour de $500 \mu$ ). Il est accolé à la paroi interne de l'utérus, présentant une solution de continuité avec l'entrée des canaux des spermathèques, si bien qu'en l'extrayant de l'utérus, on provoque obligatoirement une brèche dans sa paroi, par où s'écoule le sperme (photo $\mathrm{n}^{\circ} 2$ ).
En essayant de définir une chronologie de son élaboration, par de multiples dissections des deux sexes au cours de l'accouplement, on s'aperçoit que le «spermatophore » n'est constitué qu'à la toute dernière période du rapprochement sexuel, le mâle ne se retirant qu'après son introduction dans l'utérus de la femelle. Il est donc inutile de chercher sa présence dans les voies génitales femelles avant que l'accouplement ait cessé.

Le hasard nous a mis plusieurs fois en présence du «spermatophore » en ouvrant les genitalia du mâle avant que l'accouplement ne soit consommé, mais aux derniers instants seulement (photo $\mathrm{n}^{\circ} 3$ ). Il est donc certain que ce spermatophore est élaboré par le mâle. Les sécrétions des glandes annexes du tractus génital mâle contribuent de toute évidence à sa constitution : les grosses cellules polygonales de ces glandes sont le siège de très fortes flaques de sécrétion, occupant la totalité de leur cytoplasme à la phase ultime de l'accouplement.

Nous ne discuterons pas ici de l'intérêt phylogénique d'un tel processus dans le transport du sperme chez Glossina. Il s'agit d'une simple note destinée à confirmer l'observation de J. N. POLLOCK. Une étude plus détaillée de la question est reproduite dans un diplôme d'études approfondies du troisième cycle 


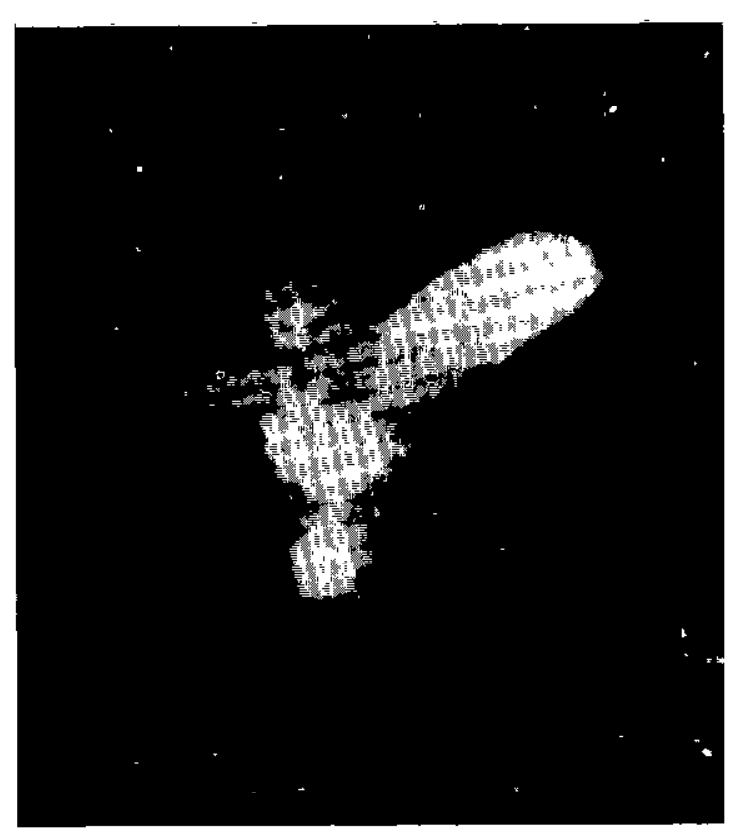

Photo $\mathrm{n}^{\mathrm{o}} 1$

Spermatophore dégagé de l'utérus chez Glossina austeni Newstead.

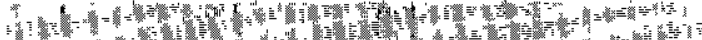
1) ;

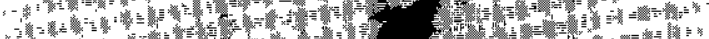
5. M. (4) 1 WW W W WII

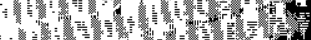
HI (1) (1) Nond

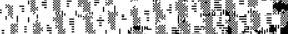

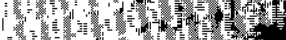
W. 1.) $(1)$ W (1) Win 1) 1 W in 1 , (v)

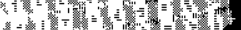
1., (1) 1. (n)

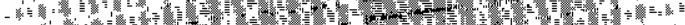
(1) : (1)

Photo no 2

Glossina austeni Newstead: Spermatophore extrait de l'utérus.

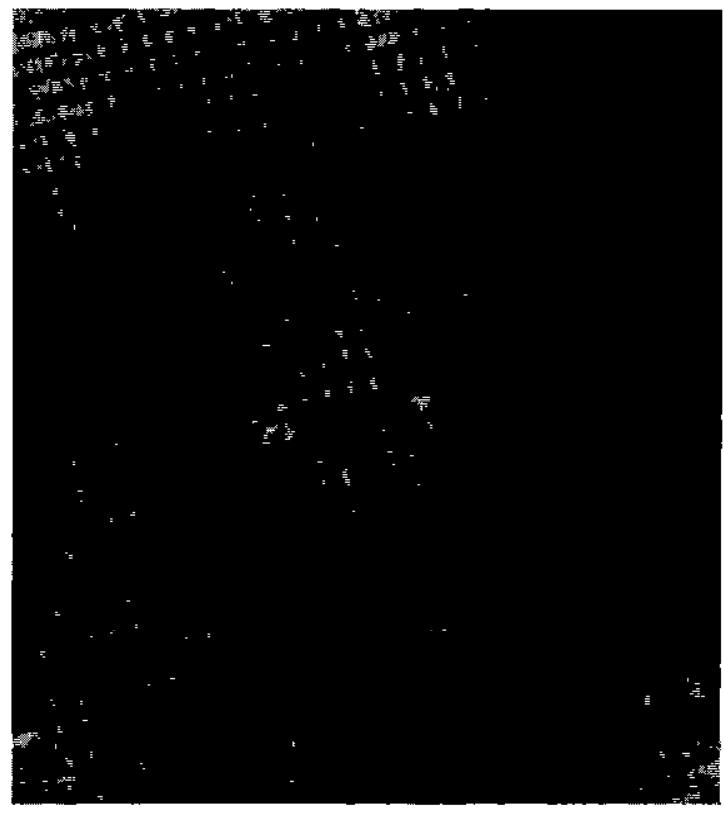

Photo no 3

Glossina morsitans Westwood. Spermatophore en voie d'expulsion chez le mâle.

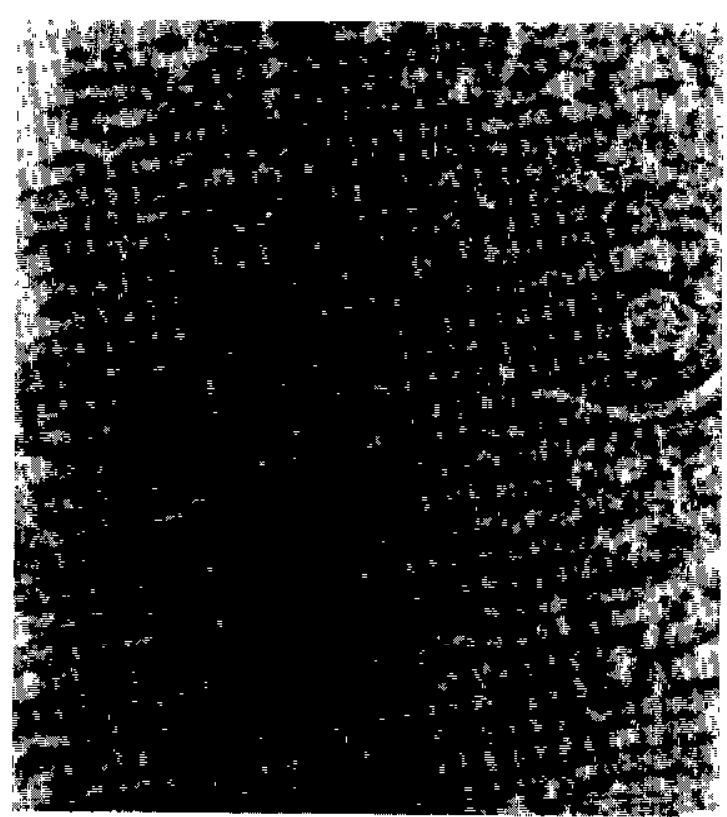

Photo no 4

Glossina morsitans Westwood. Flaques de sécrétion des glandes accessoires du tractus génital mâle. 
(D.E.A.) à la Faculté des Sciences de Paris et sera publiée ultérieurement dans la Revue

d'Elevage et de Médecine vétérinaire des Pays tropicaux.

\section{BIBLIOGRAPHIE}

POLLOCK (J. N.), «Sperm transfer by spermatophores in Glossina austeni Newstead», Nature, $1970,225,1063-1064$.

\section{SUMMARY \\ Note on some aspects of insemination in Glossina (diptera-muscidae).}

Some precisions are given that confirm the observation made by J. N. POLLOCK on Glossina austeni Newstead. During the mating, the sperm transfer occurs through a spermatophore which is built in the male before to be introduced in the uterus of the female.

\section{RESUMEN}

Nota sobre algunos aspectos de la inseminación en las glossinas (dipteramuscidae).

Se dan algunas precisiones que confirman la observación hecha por J. N. POLLOCK sobre Glossina austeni Newstead: durante el acoplamiento el trasporte de la esperma se hace mediante un espermatoforo que se constituye en el macho antes de estar introducido en el útero de la hembra. 